\title{
Nightmares in People with COVID-19: Did Coronavirus Infect Our Dreams?
}

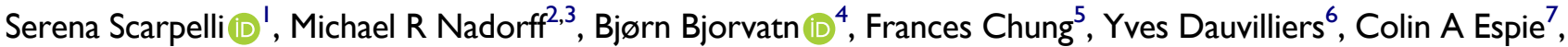 \\ Yuichi Inoue $^{8}$, Kentaro Matsui ${ }^{9,10}$, llona Merikanto $\mathbb{D}^{11,12}$, Charles M Morin ${ }^{13}$, Thomas Penzel $\mathbb{D}^{14}$, \\ Mariusz Sieminski $\mathbb{D}^{15}$, Han Fang $\mathbb{D}^{16}$, Tainá Macêdo $\mathbb{D}^{17}$, Sérgio A Mota-Rolim ${ }^{18}$, Damien Leger $\mathbb{D}^{19}$, \\ Giuseppe Plazzi $\mathbb{D}^{20,21}$, Ngan Yin Chan ${ }^{22}$, Markku Partinen ${ }^{23}$, Courtney J Bolstad (D) ${ }^{2}$, Brigitte Holzinger (D) ${ }^{24,25}$, \\ Luigi De Gennaro (iD) 1,26
}

\begin{abstract}
'Department of Psychology, Sapienza University of Rome, Rome, Italy; ${ }^{2}$ Mississippi State University, Mississippi State, MS, USA; ${ }^{3}$ Department of Psychiatry and Behavioral Sciences, Baylor College of Medicine, Houston, TX, USA; ${ }^{4}$ Department of Public Health and Primary Care, University of Bergen and Norwegian Competence Center for Sleep Disorders, Haukeland University Hospital, Bergen, Norway; ${ }^{5}$ Department of Anesthesiology and Pain Medicine, University Health Network, University of Toronto, Toronto, Canada; 'leep-Wake Disorders Unit, Department of Neurology, Guide-Chauliac Hospital, CHU Montpellier, INM, Univ Montpellier, INSERM, Montpellier, France; ${ }^{7}$ Sleep and Circadian Neuroscience Institute, Nuffield Department of Clinical Neurosciences, University of Oxford, Oxford, UK; ${ }^{8}$ Tokyo Medical University, Tokyo, Japan; ${ }^{9}$ Department of Laboratory Medicine, National Center Hospital, National Center of Neurology and Psychiatry, Kodaira, Japan; ${ }^{10}$ Department of Psychiatry, Tokyo Women's Medical University, Tokyo, Japan; "'SleepWell Research Program, Faculty of Medicine, University of Helsinki, Helsinki, Finland; ${ }^{12}$ Department of Public Health Solutions, Finnish Institute for Health and Welfare, Helsinki, Finland; ${ }^{13}$ École de Psychologie, Centre d'étude des troubles du sommeil, Centre de recherche CERVO/Brain Research Center, Université Laval, Québec, Canada; ${ }^{14}$ Sleep Medicine Center, Charité Universitätsmedizin Berlin, Berlin, Germany; ${ }^{15}$ Department of Emergency Medicine, Medical University of Gdansk, Gdansk, Poland; ${ }^{16}$ Department of Pulmonary and Critical Care Medicine, Peking University People's Hospital, Beijing, People's Republic of China; ${ }^{7}$ Department of Psychology, Federal University of Rio Grande do Norte, Natal, Brazil; ${ }^{18}$ Brain Institute, Physiology and Behavior Department, and Onofre Lopes University Hospital - Federal University of Rio Grande do Norte, Natal, Brazil; ${ }^{19}$ Université de Paris, APHP, Hôtel-Dieu, Centre du Sommeil et de la Vigilance, Paris, France; ${ }^{20}$ IRCCS, Istituto delle Scienze Neurologiche di Bologna, Bologna, Italy; ${ }^{21}$ Department of Biomedical, Metabolic and Neural Sciences, University of Modena and Reggio Emilia, Modena, Italy; ${ }^{22} \mathrm{Li}$ Chiu Kong Family Sleep Assessment Unit, Department of Psychiatry, Faculty of Medicine, The Chinese University of Hong Kong, Shatin, Hong Kong, Special Administrative Regions, People's Republic of China; ${ }^{23}$ Helsinki Sleep Clinic, Terveystalo Healthcare, and Department of Neurosciences, Clinicum, University of Helsinki, Helsinki, Finland; ${ }^{24}$ Institute for Consciousness and Dream Research, Vienna, Austria; ${ }^{25}$ Medical University Vienna, Postgraduate Master Program Medical Sleep Coaching, ZK-Schlafcoaching, Vienna, Austria; ${ }^{26}$ IRCCS Fondazione Santa Lucia, Rome, Italy
\end{abstract}

Correspondence: Luigi De Gennaro, Tel +39-06-49917647, Fax +39-06-499|77II, Email luigi.degennaro@uniromal.it

Introduction: A growing number of studies have demonstrated that the coronavirus disease-19 (COVID-19) pandemic has severely affected sleep and dream activity in healthy people. To date, no investigation has examined dream activity specifically in COVID-19 patients.

Methods: As part of the International COVID-19 Sleep Study (ICOSS), we compared 544 COVID-19 participants with 544 matchedcontrols. A within-subjects comparison between pre-pandemic and pandemic periods computed separately for controls and COVID-19 participants were performed on dream recall and nightmare frequency (DRF; NF). Also, non-parametric comparisons between controls and COVID-19 participants were carried out. Further, we compared psychological measures between the groups collected during pandemic. Ordinal logistic regression to detect the best predictors of NF was performed.

Results: We found that people reported greater dream activity during the pandemic. Comparisons between controls and COVID-19 participants revealed a) no difference between groups concerning DRF in the pre-pandemic period and during the pandemic; b) no difference between groups concerning nightmare frequency in the pre-pandemic period; and c) COVID-19 participants reported significantly higher NF than controls during pandemic $(\mathrm{p}=0.003)$. Additionally, we showed that a) anxiety, depression, post-traumatic stress-disorder (PTSD) symptom scores were higher in COVID-19 participants than controls; and b) quality of life and health as well as wellbeing (WHO-5) scores were significantly higher in controls than COVID-19 participants. Finally, ordinal logistic regression indicates that DRF $(p<0.001)$, PTSD $(p<0.001)$, anxiety $(p=0.018)$, insomnia $(p=0.039)$, COVID-19 severity ( $p=0.014)$, sleep duration $(\mathrm{p}=0.003)$ and age $(\mathrm{p}=0.001)$ predicted NF.

Discussion: Our work shows strong associations between increased nightmares in those reporting having had COVID-19. This suggests that the more that people were affected by COVID-19, the greater the impact upon dream activity and quality of life.

Keywords: dreaming, pandemic, sleep, PTSD, anxiety, COVID-19 severity

Received: 21 October 2021 Accepted: 1 January 2022

Published: 24 January 2022 


\section{Introduction}

In March 2020, the World Health Organization (WHO) proclaimed the novel Coronavirus disease outbreak (COVID-19) to be a global pandemic. COVID-19 is characterized by a severe acute respiratory syndrome and much clinical and research attention has been paid to finding an effective treatment or prevention strategy to defeat the virus.

In the last year, an impressive number of studies has focused on the psychological consequences of the pandemic both on the worldwide population ${ }^{1}$ and infected people.,

In particular, a growing literature has highlighted that the COVID-19 pandemic strongly affected Sleep. ${ }^{4-9}$ Among the general population, cross-sectional investigations found dysregulation in sleep-wake schedules ${ }^{7,9,10}$ and insomnia symptoms. ${ }^{11-13}$ Additionally, a recent review emphasized that COVID-19 patients have higher prevalence rates of sleep disturbances. ${ }^{12}$ On the one hand, the fear related with the virus may be one of the main reasons for poor sleep quality. On the other hand, it should be noted that the core symptoms of the infection such as fever, coughing, breathing difficulties and physical pain, are highly correlated with sleep, problems. ${ }^{14-16}$ Moreover, hospitalization (pharmacological treatments for the infection could contribute to sleep disruption). ${ }^{16,17}$

In parallel with sleep problems, several studies show that the pandemic is associated with alterations of dreams and nightmares among healthy people. ${ }^{18-25}$

From a qualitative point of view, healthy individuals have reported higher negative emotional intensity in dream content during the COVID-19 outbreak. ${ }^{20,21,24,26}$ Moreover, pandemic-related contents were identified both during the first wave of pandemic ${ }^{20,27-29}$ and in the post-lockdown period. ${ }^{25}$ In a relatively large United States sample $(\mathrm{N}=3031)$, dream activity and mental health were shown to be associated during the pandemic, with the finding that the more participants were affected by the pandemic, the more it affected their dreams. ${ }^{27}$ Similarly, individuals having COVID-19related traumatic experiences, such as death or disease of relatives/friends, report increased distress in their dream content ${ }^{20}$ and nightmares. ${ }^{24}$ Further, people reporting more changes in their life situation (eg, sleep habits or working life) have more emotional dreams. ${ }^{24}$ Furthermore, some trait-like features have an impact on oneiric activity. Indeed, women have shown higher DRF than men, ${ }^{18,20,22,24,26}$ and older adults have reported lower (dream recall frequency) $\mathrm{DRF}^{22,24}$ and (nightmare frequency) $\mathrm{NF}^{24}$ than younger individuals.

Importantly, sleep patterns and oneiric activity appear to be strictly related since poor sleep quality predicts high DRF and NF during the lockdown. ${ }^{21,24}$ Also, a longitudinal study revealed a greater lucid dream frequency during lockdown than post-lockdown. ${ }^{25}$

An international investigation highlighted that sleep disturbances and parasomnia-like events such as sleep talking and REM sleep behavior disorder symptoms are associated with high DRF. ${ }^{14}$ Further, psychological variables influence the quantitative features of oneiric activity since Post-Traumatic Stress Disorder (PTSD) symptoms are related to DRF, ${ }^{22}$ and both anxiety ${ }^{24}$ and depression ${ }^{21,24}$ are associated with high NF.

Although the literature evidenced the high proportion of sleep disturbances among COVID-19 patients, ${ }^{3,12}$ to the best of our knowledge, we have no information on the oneiric activity of people with COVID-19 and no study has compared the oneiric activity of those who have had COVID-19 and those who have not. This research is crucial for several reasons. First, we are beginning to understand some of the long-term consequences of the virus including physical, ${ }^{30,31}$ cognitive, ${ }^{32,33}$ and mental health ${ }^{34}$ changes. Given these changes, it is very likely that there are notable differences in sleep and dreaming that come subsequently to infection. Importantly, nightmares have been linked with many forms of psychopathology such as anxiety and depression, ${ }^{35}$ borderline personality disorder, ${ }^{36}$ and suicidal behavior and mortality, ${ }^{37}$ even when accounting for the effects of post-traumatic stress disorder and related Disorders. ${ }^{38-40}$ Thus, the development of nightmares is clinically relevant.

To fill this notable gap in the literature, we aimed to investigate the relationship between COVID-19 and oneiric activity. Since participants who experienced greater life changes due to COVID-19 pandemic had more disturbing dreams or contents related to the disease outbreak, ${ }^{20,24,27}$ we predicted that individuals who have had COVID-19 would report greater changes in their dreams than those without COVID-19, especially concerning nightmares. Second, we hypothesized that COVID-19-related factors, such as the illness severity and quarantine duration, would be associated with participants' nightmares. 


\section{Materials and Methods \\ Study Design and Participants}

The current research protocol is part of a wider project by the International COVID-19 Sleep Study (ICOSS). ${ }^{41}$ Fourteen countries/regions including Austria, Brazil, Canada, Hong Kong, Province Jilin (China), Finland, France, Italy, Japan, Norway, Sweden, Poland, the UK, and the USA were involved in the cross-sectional study. Each participant filled out a web-survey between May and July 2020. Different web-platforms, (eg, REDcap, Qualtrics) were used to distribute the online-survey, which was translated into the national language of each country. To recruit a large number of participants, the web-survey was promoted via university communication systems, newspapers, social-media platforms, newsletters to hospitals, and through sleep societies' websites.

Investigators from each country obtained ethical approval from the local ethics committee, if needed according to local legislations. The study was approved by the Institutional Ethics Committee of the Department of Psychology of "Sapienza" University of Rome and was conducted in accordance with the Declaration of Helsinki (protocol number: 0000836, May 23rd, 2020). Subjects had to be at least 18 years of age and agreed voluntarily and anonymously to take part in the survey. All participants provided electronic informed consent before accessing the web-survey. General data protection regulations were applied to ensure privacy and confidentiality.

Data from 544 participants declaring they have had COVID-19 (they answered "yes" to the question: "Have you had COVID-19?") and 544 matched-controls were extracted from respondents (25,500 participants) and included in the current investigation. The control group was matched according to the following criteria: age, gender, country, ethnicity, type of residential area, marital status, education, work, quarantine duration. Both asymptomatic and symptomatic subjects with different degrees of infection severity were included in the COVID-19 group.

Other data concerning the whole sample and analyzed with different aims have been reported elsewhere. ${ }^{13,22,42}$

\section{Instruments}

The web-survey included a section collecting sociodemographic information (age, gender, ethnicity, country, residential area, marital status, education, work) and COVID-19-related information (infection, severity, confinement duration). Also, information about hypnotic's usage and financial burden were collected.

The questionnaires used to assess psychological dimensions are described below:

1. Generalized Anxiety Disorder-2 (GAD-2). ${ }^{43}$ A 2-item short form of the GAD-7 evaluated anxiety on scales of 0 to 3. A score of three is the recommended cut-off for detecting significant anxiety symptoms.

2. Patient Health Questionnaire-2 (PHQ-2). ${ }^{43}$ A 2-item abbreviated version of the PHQ-9 assessed depression disturbances on scales of 0 to 3 . A score of 3 is the cut-off for identifying clinically relevant symptoms of depression.

3. Stress. ${ }^{44}$ A single item (1-5 rating) evaluated the current stress from "not at all" to "very much".

4. Post-traumatic stress disorder. ${ }^{45}$ Two items were used to assess the key symptoms of PTSD. Respondents rated their symptoms on scales of 1 to 5 .

5. Well-Being Index (WHO-5). ${ }^{46} \mathrm{~A}$ short questionnaire of 5 statements to assess the overall psychological wellbeing. In relation to the past two weeks, respondents rated each item on $0-5$ scales. The raw score is multiplied by four, resulting in a total score from 0 to 100 . Higher scores indicate a better quality of life.

6. Quality of life and health. ${ }^{47}$ Two items using 0-100 linear visual analog scales measured the quality of life and quality of health.

The questionnaires used to assess sleep and dream variables are described below:

1. Basic Nordic sleep questionnaire. ${ }^{48}$ This instrument assessed sleep onset and sleep maintenance problems, early morning awakenings, use of hypnotics, sleepiness and fatigue. Respondents rated each item on a scale from 1 to 5 , based on how many nights per week sleep problems occurred (1= "Never or less frequently than once per month", 
$2=$ "Less frequently than once per week", $3=$ "1-2 nights per week", $4=$ "3-5 nights per week", $5=$ "Nightly or almost nightly"). Similarly, two items were added to assess dream recall frequency and nightmares. Sleep quality was evaluated on a scale from 1 (well) to 5 (badly). All statements were rated "during the pandemic" and "before the pandemic". Specifically, the questionnaire required to answer each question referring to the COVID-19 emergency (ie, pandemic period) and the period immediately preceding the time of confinement/restrictions/social isolation (ie, pre-pandemic period).

2. Insomnia Severity Index (ISI). ${ }^{49}$ A 7 -item questionnaire was used to evaluate the severity of nighttime and daytime symptoms of insomnia during the past week before answering the survey, rated on a scale of 0 to 4 . A total score of 0-7 indicates no insomnia, 8 to 14 subthreshold clinical insomnia, 15 to 21 insomnia of moderate severity, and 22 and above indicates severe insomnia.

Specifically, the following variables were extracted: the wellbeing (WHO-5) score, the quality of life, and quality of health measures; the GAD and PHQ measures dichotomized accordingly to their cut-off (3); ${ }^{43}$ the stress score dichotomized considering cut-off $=3$; the ISI score dichotomized accordingly to its cut-off for clinical insomnia (15); ${ }^{49}$ the DRF; the NF; sleep quality categorized as good (well, rather well, neither well nor badly) and poor (rather badly, badly); the sleep duration categorized as short ( $<6$ hours), normal (6-8 hours), and long ( $\geq 9$ hours) $;{ }^{50,51}$ the PTSD measures dichotomized as low risk $(<3)$ and high risk $(\geq 3)$. Also, among socio-demographic and COVID-19 related variables were considered: age; gender; quarantine duration categorized as $\leq 1$ month and $>1$ month; self-reported COVID-19-severity categorized as low (no symptoms or mild) and high (moderate or severe symptoms and/or life threatening).

The selection of dependent measures was based on (a) the findings of other studies published during pandemic ${ }^{18-28}$ and (b) the need to avoid any overlapping (ie, duplication of results) with results of other publications of the ICOSS group. ${ }^{13,22,42}$ The complete protocol is available on Partinen et al. ${ }^{41}$

\section{Statistical Analysis}

The statistical procedures were carried out using the Statistical Package for Social Sciences (sPss) version 25.0 and MATLAB R2019. First, descriptive analyses were carried out to outline the sociodemographic characteristics of the COVID-19 participants and the control group considering the following features: age, gender, ethnicity, residential area, marital status, living alone, education, and work status.

To assess the differences concerning oneiric activity, non-parametric within comparisons (Wilcoxon test) between prepandemic and pandemic periods were computed separately for controls and COVID-19 participants. Further, non-parametric comparisons (Mann-Whitney test) between controls and COVID-19 participants were performed on DRF and NF.

Also, for a descriptive purpose, we compared psychological measures (wellbeing, quality of life, quality of health, symptoms of anxiety, depression, stress, and PTSD) between the two groups. Data on insomnia were not included in these analyses, since this comparison in people with or without COVID has been reported elsewhere. ${ }^{13}$

According to the hypothesis of the study, an ordinal logistic regression analysis in the COVID-19 group was performed to explore the role of psychological variables, sleep measures, quarantine duration, and COVID-19severity on nightmare frequency (dependent variable). Specifically, the following predictors have been included: PTSD-repeated disturbing thoughts/memories (low risk, high risk), anxiety (yes, no), depression (yes, no), stress (yes, no), DRF (from 1 to 5), sleep quality (good, poor), sleep duration (long, normal, short), insomnia (yes, no), quality of life, quality of health, wellbeing, quarantine duration ( $\leq 1$ month, $>1$ month), COVID-19 severity (low, high). Considering the well-known effect of age and gender on dreaming, ${ }^{22,24}$ we adjusted the model for these two variables. We entered the variables simultaneously into a single model. Multicollinearity between the independent variables was assessed before running the logistic regression by calculating Variance Inflation Factors (VIF). The VIF statistics for all variables included in the regression model were under three, indicating only moderate correlation. 


\section{Results}

\section{Characteristics of Samples}

The characteristics of participants are shown in Table 1. Data from 1088 subjects revealed that the majority of participants were female (about $60 \%$ in both groups). The most represented age range was 34-44 years (27-28\%). Concerning ethnicity, participants were mostly Caucasian (controls: 63.1\%; COVID-19 participants: 60.9\%) and nearly $25 \%$ of samples in both groups came from the USA. More than $80 \%$ of the subjects in both groups resided in urban areas. Most of the individuals were married/cohabiting both among controls (59.2\%) and COVID-19 individuals (60.4\%). Respondents mainly were graduates with a bachelor's degree both among controls (41.9\%) and COVID-19 participants (39.7\%). More than half of the subjects had a regular day work in both groups. Also, nearly $40 \%$ of participants had a forced quarantine lasting more than a month in both groups. Approximately $70 \%$ of controls and $60 \%$ of COVID-19 participants reported using hypnotics "never or less frequently than once per month". Finally, just a small percentage of individuals (controls: 4.4\%; COVID-19 participants: 6.8\%) reported a high degree of financial burden during the pandemic.

Descriptive data on dream variables (DRF and NF) across different countries were reported in Figures S1 and $\underline{\mathrm{S} 2}$.

\section{Differences in the Oneiric Activity: Controls versus COVID-19 Participants}

First, we explored how the two groups were different concerning the variables related to oneiric activity. The withincomparisons (Wilcoxon test) between pre-pandemic and pandemic periods (Table S1), computed separately for controls and COVID-19 participants, revealed significant differences $(p<0.001)$ in DRF and nightmare frequency (Figure 1), highlighting that during pandemic people reported greater oneiric activity.

Crucially, non-parametric comparisons (Mann-Whitney test) between controls and COVID-19 participants (Table S2), revealed a) no difference between groups concerning DRF in the pre-pandemic period and during pandemic; b) no difference between groups concerning NF in the pre-pandemic period; and c) COVID-19 participants reported significantly higher NF than controls during pandemic $(p=0.003$; Figure 2$)$.

\section{Comparisons for Clinical Variables During Pandemic}

Second, we then assessed whether the two groups differ in terms of some psychological and clinical variables during pandemic. The comparisons between the control group and the COVID-19 group showed that: a) symptoms of anxiety $(p=0.001)$, depression $(p=0.001)$, PTSD (repeated disturbing thoughts/memories, $p=0.003$ and feeling very upset about past, $p=0.002)$ scores were higher in COVID-19 participants than controls; and $\mathrm{b}$ ) quality of life $(p=0.005)$ and quality of health $(p<0.001)$, as well as Wellbeing (WHO-5) scores $(p=0.002)$ were significantly higher in controls than COVID-19 participants (Table 2).

\section{Predictors of Nightmare Frequency in COVID-19 Participants}

Third, we have determined the best predictor variables for the most robust variable associated with dream activity that discriminated the two groups. To this aim, ordinal logistic regression model was computed on 499 COVID-19 participants (after excluding subjects with missing values). The outcome provided a significant model: likelihood ratio: chi-squared $=286.50, p<0.001$; Negelkerke's $\mathrm{R}^{2}=0.46$.

The analysis revealed that higher NF was associated with a) DRF (daily or almost daily: Odds Ratio $[\mathrm{OR}]=19.26$, 95\% Confidence Interval $[\mathrm{CI}]: 9.38,39.57$; $3-5$ days per week: $\mathrm{OR}=11.54,95 \% \mathrm{CI}$ : $6.01,22.14 ; 1-2$ days per week: $\mathrm{OR}=9.67,95 \% \mathrm{CI}: 5.11,18.31$; less frequently than once per week: $\mathrm{OR}=2.82,95 \% \mathrm{CI}: 1.47,5.41$ ); b) PTSD symptoms $(\mathrm{OR}=2.58,95 \% \mathrm{CI}: 1.67,3.96)$; c) anxiety symptoms $(\mathrm{OR}=1.76,95 \% \mathrm{CI}: 1.10,2.81)$; d $)$ insomnia symptoms $(\mathrm{OR}=1.63,95 \% \mathrm{CI}: 1.02,2.60)$; e) COVID-19 severity $(\mathrm{OR}=1.60,95 \% \mathrm{CI}: 1.10,2.34)$; f) sleep duration (long: $\mathrm{OR}=0.43,95 \% \mathrm{CI}: 0.25,0.75$; normal: $\mathrm{OR}=0.55,95 \% \mathrm{CI}: 0.35,0.87)$ and g) age $(55-64$ years: $\mathrm{OR}=0.29$, $95 \%$ CI: $0.14,0.59 ; 45-54$ years: $\mathrm{OR}=0.36,95 \%$ CI: $0.20,0.66)$. Specifically, having higher DRF, high anxiety and insomnia symptoms, high risk of PTSD, lower sleep duration and younger age predict higher NF. The results are reported in Table 3. 
Table I Characteristics of Participants

\begin{tabular}{|c|c|c|}
\hline & Controls N (\%) & COVID-I 9 Participants N (\%) \\
\hline \multicolumn{3}{|c|}{$\begin{array}{l}\text { Gender } \\
\text { (C: } n=544 ; C P: n=544)\end{array}$} \\
\hline Male & $219(40.3)$ & $215(39.5)$ \\
\hline Female & $325(59.7)$ & $329(60.5)$ \\
\hline \multicolumn{3}{|c|}{$\begin{array}{l}\text { Age, years } \\
(C: n=544 ; C P: n=544)\end{array}$} \\
\hline$<25$ & $81(14.9)$ & $81(14.9)$ \\
\hline $25-34$ & $102(18.7)$ & $100(18.4)$ \\
\hline $35-44$ & $149(27.4)$ & $154(28.3)$ \\
\hline $45-54$ & $99(18.2)$ & $99(18.2)$ \\
\hline $55-64$ & $64(11.8)$ & $64(11.8)$ \\
\hline $65+$ & $49(9)$ & $46(8.5)$ \\
\hline \multicolumn{3}{|c|}{$\begin{array}{l}\text { Ethnicity } \\
\text { (C: } n=544 ; C P: n=539)\end{array}$} \\
\hline Caucasian & $343(63.1)$ & $328(60.9)$ \\
\hline Asian & $7 \mid(\mid 3.1)$ & $74(13.7)$ \\
\hline African & $56(10.3)$ & $60(11.1)$ \\
\hline Hispanic & $4 \mid(7.5)$ & $46(8.5)$ \\
\hline Other & $33(6.1)$ & $31(5.8)$ \\
\hline \multicolumn{3}{|c|}{$\begin{array}{l}\text { Country } \\
\text { (C: } n=544 ; C P: n=544)\end{array}$} \\
\hline Austria & $9(1.7)$ & $9(1.7)$ \\
\hline Brazil & $76(14.0)$ & $87(16.0)$ \\
\hline Canada & $42(7.7)$ & $35(6.4)$ \\
\hline China, Hong Kong & $2(0.4)$ & $2(0.4)$ \\
\hline China, Jilin & II (2.0) & $10(1.8)$ \\
\hline Finland & $21(3.9)$ & $23(4.2)$ \\
\hline France & $37(6.8)$ & $33(6.1)$ \\
\hline Italy & $26(4.8)$ & $25(4.6)$ \\
\hline Japan & $61(11.2)$ & 63 (11.6) \\
\hline Norway & $17(3.1)$ & $21(3.9)$ \\
\hline Poland & $3(0.6)$ & $4(0.7)$ \\
\hline Sweden & $59(10.8)$ & $50(9.2)$ \\
\hline UK & $43(7.9)$ & $46(8.5)$ \\
\hline USA & $137(25.2)$ & $136(25.0)$ \\
\hline
\end{tabular}

(Continued) 
Table I (Continued).

\begin{tabular}{|c|c|c|}
\hline & Controls N (\%) & COVID-I9 Participants N (\%) \\
\hline \multicolumn{3}{|l|}{$\begin{array}{l}\text { Residential area } \\
(C: n=543 ; C P: n=54 I)\end{array}$} \\
\hline Rural & $98(18.1)$ & 91 (16.8) \\
\hline Urban & $445(81.9)$ & $450(83.2)$ \\
\hline \multicolumn{3}{|l|}{$\begin{array}{l}\text { Marital status } \\
(C: n=544 ; C P: n=543)\end{array}$} \\
\hline Single & $184(33.8)$ & $|7|(3 \mid .5)$ \\
\hline Married/cohabiting & $322(59.2)$ & $328(60.4)$ \\
\hline Divorced/separated & $29(5.3)$ & $35(6.4)$ \\
\hline Widowed & $9(1.7)$ & $9(1.7)$ \\
\hline \multicolumn{3}{|l|}{$\begin{array}{l}\text { Education } \\
(C: n=544 ; C P: n=543)\end{array}$} \\
\hline Primary & $7(1.3)$ & $9(1.7)$ \\
\hline Secondary & $58(10.7)$ & $67(12.3)$ \\
\hline Vocational & $45(8.3)$ & $47(8.7)$ \\
\hline Bachelor & $228(41.9)$ & $216(39.7)$ \\
\hline Master & $164(30.1)$ & $160(29.5)$ \\
\hline Doctor & $42(7.7)$ & $44(8.1)$ \\
\hline \multicolumn{3}{|l|}{$\begin{array}{l}\text { Work } \\
(C: n=539 ; \text { CP: } n=539)\end{array}$} \\
\hline Regular day work & $305(56.6)$ & $283(52.5)$ \\
\hline Irregular day work & $48(8.9)$ & $52(9.7)$ \\
\hline Student & $59(10.9)$ & $47(8.7)$ \\
\hline Shift/night work & $30(5.6)$ & $58(10.8)$ \\
\hline At home, no salary & $19(3.5)$ & $20(3.7)$ \\
\hline Temporary laid off & $15(2.8)$ & $18(3.3)$ \\
\hline Unemployed & $28(5.2)$ & $26(4.8)$ \\
\hline Retired & $30(5.6)$ & $26(4.8)$ \\
\hline Lost job due to pandemic & $5(0.9)$ & $9(1.7)$ \\
\hline \multicolumn{3}{|l|}{$\begin{array}{l}\text { Quarantine duration } \\
\text { (C: } n=527 ; C P: n=537)\end{array}$} \\
\hline$\leq \mathrm{I}$ month & $302(57.3)$ & $320(59.6)$ \\
\hline$>$ I month & $225(42.7)$ & $217(40.4)$ \\
\hline $\begin{array}{l}\text { Hypnotics usage } \\
(C: n=544 ; C P: n=544)\end{array}$ & & \\
\hline
\end{tabular}


Table I (Continued).

\begin{tabular}{|c|c|c|}
\hline & Controls N (\%) & COVID-I 9 Participants N (\%) \\
\hline Never or less frequently than once per month & $389(7 \mid .5)$ & $342(62.9)$ \\
\hline Less frequently than once per week & $52(9.6)$ & $4 \mid(7.5)$ \\
\hline I-2 nights per week & $33(6.1)$ & $75(13.8)$ \\
\hline $3-5$ nights per week & $33(6.1)$ & $43(7.9)$ \\
\hline Every night or almost every night & $37(6.8)$ & $43(7.9)$ \\
\hline \multicolumn{3}{|l|}{$\begin{array}{l}\text { Financial burden } \\
(C: n=544 ; C P: n=544)\end{array}$} \\
\hline Not at all & $221(40.6)$ & $172(31.6)$ \\
\hline A little & $159(29.2)$ & $132(24.3)$ \\
\hline Somewhat & $98(18)$ & $115(21.1)$ \\
\hline Much & $42(7.7)$ & $88(16.2)$ \\
\hline Very much & $24(4.4)$ & $37(6.8)$ \\
\hline
\end{tabular}

Abbreviations: C, controls; CP, COVID-19 participants.

\section{Discussion}

To date, this is the first study investigating oneiric activity in subjects who have had COVID-19 in comparison to controls. First, consistent with previous findings, we observed that both participants with and without COVID-19 had higher dream activity during the pandemic than pre-pandemic. ${ }^{21-24}$

Importantly, during the pandemic, NF was significantly higher in the COVID-19 participants than the controls, as hypothesized. This increased nightmare rate may be a consequence of traumatic experience. ${ }^{52,53}$ Coherently, we found that having repeated disturbing thoughts/memories as a PTSD symptom, as well as anxiety, were associated with greater NF.

It should be considered that our data were collected during the "first wave" of the COVID-19 pandemic when knowledge about the virus was very poor. ${ }^{54}$ The scarce or inadequate information on the disease and its treatment, as well as the social stigmatization after diagnosis, was related to fear and feeling of uncertainty, and these factors could induce PTSD-like symptoms. ${ }^{55}$

Interestingly, we revealed that high COVID-19 disease severity predicted greater NF. Hence, people who had developed a moderate/severe or life-threatening disease reported more nightmares. Although we did not have any information about the contents or "recurring nightmares", the high NF could be a symptom of PTSD, following what was likely qualified as a traumatic event. ${ }^{56} \mathrm{We}$ could speculate that the lack of contact with families or friends during forced quarantine and/or hospitalization may have induced higher PTSD symptoms and -as a consequence- higher NF. ${ }^{57}$ Separate from experiencing a potentially traumatic experience, the presence of several nightmares after the illness may also be an attempt to cope with the COVID-19-related negative emotions. Nightmares were proposed to reflect a failure in emotional regulation processes after events characterized by significant distress. ${ }^{58}$ Also, nightmares are associated with other symptoms of psychopathology and suicidal behavior independent of PTSD symptoms, ${ }^{39,40}$ suggesting that nightmares may be also having a notable impact on the participant's life.

Additionally, we found that clinical insomnia was strictly related to higher NF in COVID-19 cases. In parallel, higher sleep duration (normal or long) was associated with lower NF. Our results are consistent with the observations during the pandemic without assessing the influence of COVID-19 infection $\left(\mathrm{eg},{ }^{22,24}\right)$ and with the current literature on dreaming. ${ }^{59}$ Poor sleep, as in the case of insomnia, promotes higher oneiric frequency. ${ }^{59}$ This evidence is supported by electroencephalographic (EEG) studies reporting that fragmented sleep, higher intra-sleep wakefulness, and greater cortical 

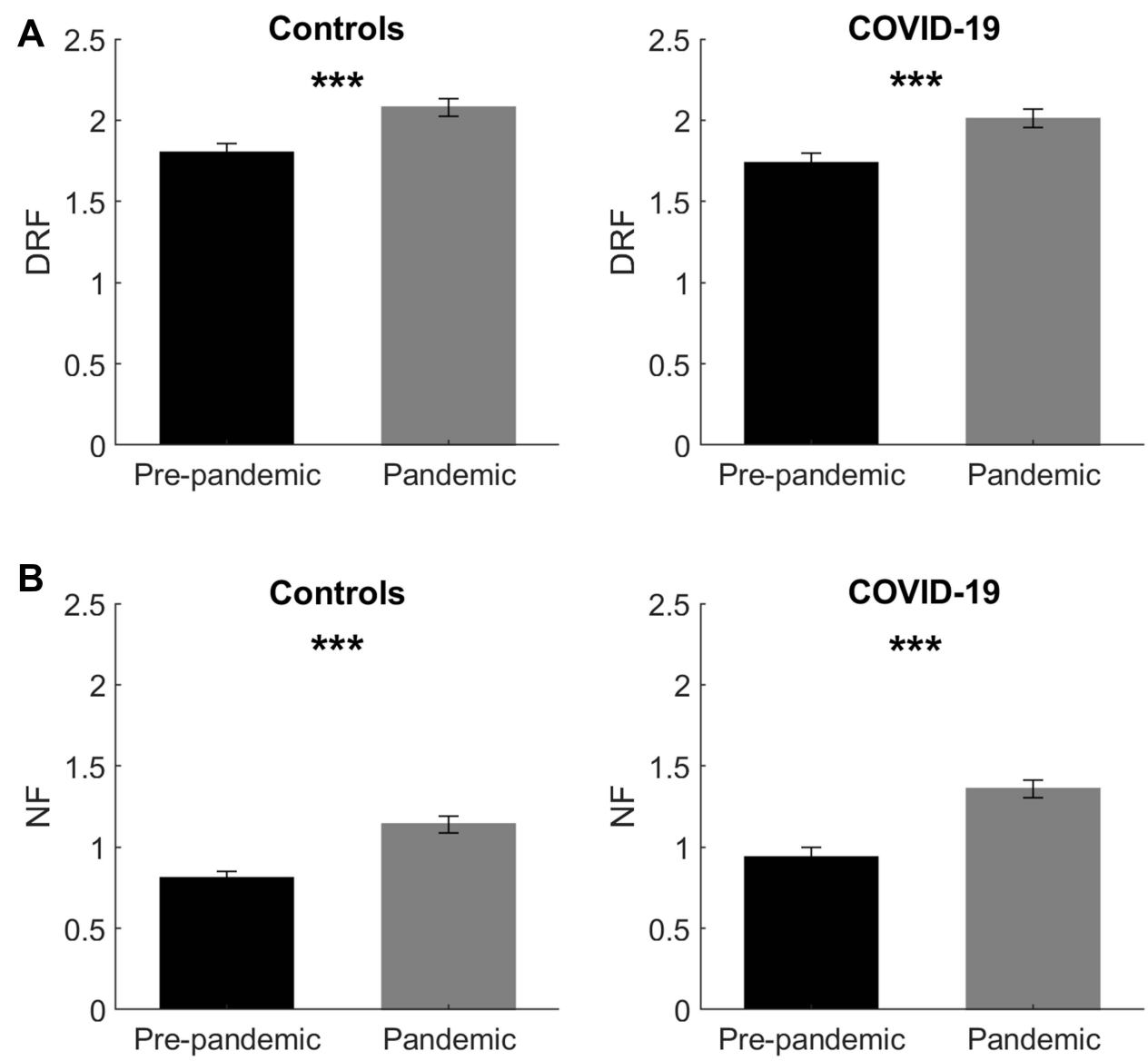

Figure I Oneiric frequency changes between pre-pandemic and pandemic period. (A) shows the results of non-parametric (within) comparisons on Dream Recall Frequency (DRF) between pre-pandemic (black bars) vs pandemic period (grey bars), performed separately for the controls and COVID-19 group. (B) depicts the results of non-parametric comparisons on Nightmare Frequency (NF) between pre-pandemic (black bars) vs pandemic period (grey bars) performed separately for the controls and COVID-19 group. Error bars represent the standard errors. Significant results are asterisked. $* * * p<0.001$.

activation are related to high DRF ${ }^{60-62}$ and - more directly - to NF. ${ }^{63-65}$ The association between insomnia and NF may also be the expression of underlying psychiatric problems, as insomnia and nightmares are both symptoms of depression and PTSD. ${ }^{66}$ Also, a previous study found that risks of insomnia were higher among COVID-19 participants. ${ }^{13}$

Accordingly, given that nightmares have been shown to be associated with many forms of psychopathology, ${ }^{35}$ the presence of nightmares may also be related to the increased psychiatric symptomatology. Indeed, COVID-19 participants had more psychiatric symptoms than controls: they reported not only greater PTSD symptoms but also more anxiety and depression. Conversely, the wellbeing measures were significantly higher in the controls. Similarly, a recent metaanalysis revealed a high prevalence of depression, anxiety, and sleep disturbances in COVID-19 patients. ${ }^{3}$ Moreover, Poyraz et $\mathrm{al}^{55}$ found that patients who had recovered from the acute COVID-19 reported not only clinically relevant PTSD but also anxiety, and depressive symptoms.

Our findings are in line with this research, with the COVID-19 participants not only experiencing increased nightmares but also significantly higher symptoms of mental disorders. Further research is warranted to delve deeper into these complex relations to determine whether either the trauma associated with having COVID-19, or the presence of nightmares may be underlying these differences in mental disorders.

Similar to the available literature on pandemic dreams, ${ }^{21,22,24}$ we confirmed that there is an "age-effect" on oneiric activity. Although nightmares and disturbing dreams in older people are scarcely investigated, ${ }^{67}$ the age-related decline of nightmares may be linked to a reduced motivation to recall dream activity during aging. ${ }^{68,69}$ 

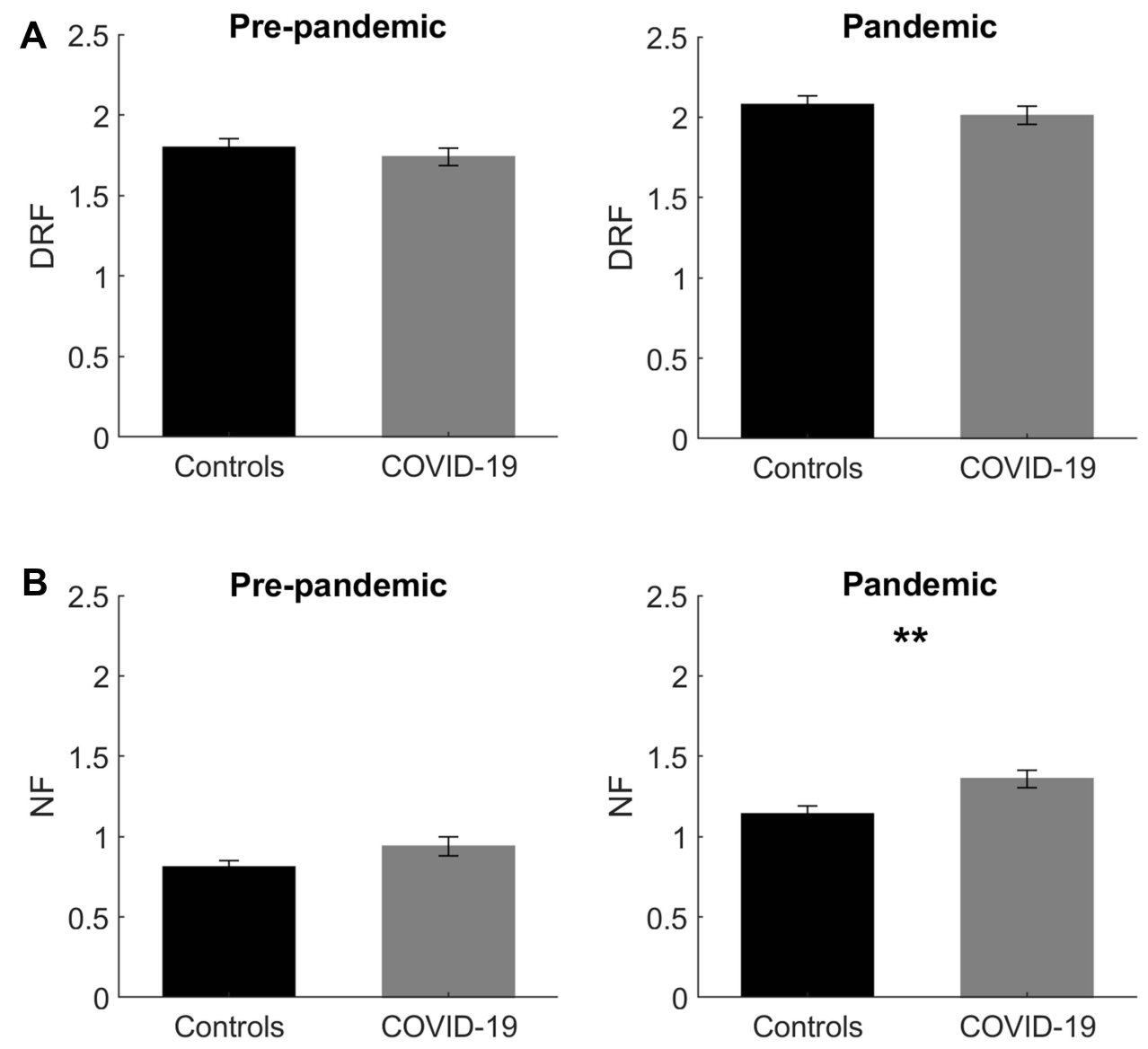

Figure 2 Oneiric frequency differences between controls and COVID-19 participants. (A) shows the results of non-parametric (between) comparisons on Dream Recall Frequency (DRF) between controls (black bars) vs COVID-19 participants (grey bars), performed separately for the pre-pandemic and pandemic period. (B) depicts the results of non-parametric comparisons on Nightmare Frequency (NF) between controls (black bars) vs COVID-19 participants (grey bars) performed separately for the prepandemic and pandemic period. Error bars represent the standard errors. Significant results are asterisked. $* * p<0.01$.

Moreover, we found that DRF and NF were associated in the COVID-19 group, and these two phenomena were also related in the wider sample examined in the previous study by the ICOSS group. ${ }^{22}$ Accordingly, other studies have also found an association between DRF and NF in the general population. ${ }^{70-72}$ Overall, considering results on the whole sample, ${ }^{22}$ it should be expected that the more people were affected by the COVID-19 pandemic, the more dream activity would also be affected. In other words, individuals' oneiric activity, in general, and nightmare frequency, more specifically, may represent a reliable index of wellbeing or discomfort. Indeed, our findings are consistent with this hypothesis, showing strong associations between increased nightmares and reduced quality of life in those reporting having had COVID-19.

A strength of the current study was that data were collected using the same methodology among different countries matching controls and infected subjects by country. However, we believe that further studies should specifically assess differences between nations since the unbalanced distribution of COVID-19 cases and the number deaths varied greatly from region to region (ie, the possibility of traumatic experiences may vary greatly) in our sample did not allow us to address this issue.

\section{Limitations}

The current investigation has some limitations. First, data collected were "double retrospective" because we required participants to give information "before" and "during" pandemic, which has an intrinsic problem of "memory bias". 73 Second, the original version of the Basic Nordic sleep questionnaire ${ }^{48}$ does not include questions regarding DRF and NF; indeed, the two questions were specially added to the questionnaire. This could be considered a limitation of our protocol since the test with these two "new" items was not standardized. Third, the information about the COVID-19 severity was 
Table 2 Mean Rank of Clinical Measures and Results of Statistical Comparisons (Mann-Whitney U-Test) Between Controls and COVID-19 Participants (Controls N=544; COVID-19 Participants= 544) During Pandemic

\begin{tabular}{|c|c|c|c|c|}
\hline & & Mean Rank & $\mathbf{Z}$ & $\mathbf{p}$ \\
\hline \multirow[t]{2}{*}{ Anxiety } & Controls & 514.07 & \multirow[t]{2}{*}{-3.24} & \multirow[t]{2}{*}{0.001} \\
\hline & COVID-19 participants & 574.93 & & \\
\hline \multirow[t]{2}{*}{ Depression } & Controls & 512.18 & \multirow[t]{2}{*}{-3.44} & \multirow[t]{2}{*}{0.001} \\
\hline & COVID-19 participants & 576.82 & & \\
\hline \multirow[t]{2}{*}{ Stress $^{\mathrm{a}}$} & Controls & 529.82 & \multirow[t]{2}{*}{-1.33} & \multirow[t]{2}{*}{0.184} \\
\hline & COVID-19 participants & 554.25 & & \\
\hline \multirow[t]{2}{*}{ PTSD: repeated disturbing thoughts/memories } & Controls & 517.32 & \multirow[t]{2}{*}{-2.95} & \multirow[t]{2}{*}{0.003} \\
\hline & COVID-19 participants & 571.68 & & \\
\hline \multirow[t]{2}{*}{ PTSD: feeling very upset about past } & Controls & 515.85 & \multirow[t]{2}{*}{-3.06} & \multirow[t]{2}{*}{0.002} \\
\hline & COVID-19 participants & 572.20 & & \\
\hline \multirow[t]{2}{*}{ Quality of Life ${ }^{b}$} & Controls & 570.63 & \multirow[t]{2}{*}{-2.80} & \multirow[t]{2}{*}{0.005} \\
\hline & COVID-19 participants & 517.32 & & \\
\hline \multirow[t]{2}{*}{ Quality of Health ${ }^{c}$} & Controls & 593.02 & \multirow[t]{2}{*}{-5.26} & \multirow[t]{2}{*}{$<0.00$} \\
\hline & COVID-19 participants & 493.08 & & \\
\hline \multirow[t]{2}{*}{ Wellbeing (WHO-5) } & Controls & 573.94 & \multirow[t]{2}{*}{-3.09} & \multirow[t]{2}{*}{0.002} \\
\hline & COVID-19 participants & 515.06 & & \\
\hline
\end{tabular}

Notes: ${ }^{\mathrm{a} C o n t r o l s}=543$, COVID- 19 participants $=540 .{ }^{\mathrm{b}}$ Controls $=544$, COVID- 19 participants $=543 .{ }^{\mathrm{c}}$ Controls $=542$, COVID-19 participants $=543$. Significant values are indicated in bold.

Abbreviation: PTSD, post-traumatic stress disorder.

self-reported and this would imply that the "severity" of the disease was not objectively evaluated by a physician/medical healthcare worker or based on objective measures.

Further, the survey did not allow collecting information about the physical and mental state of COVID-19 patients before getting the disease. This would represent a limitation since pre-existing health problems, especially mental illness, could have affected the patient's sleep and dreams. ${ }^{74}$

Also, we do not have any information about how much time has passed since infection among the COVID-19 subjects. This variable may be essential to better understand the relationship between higher nightmares and the severity of infection. We are aware that in some cases, COVID-19 symptoms may still persist at the time of the survey. In other words, participants may have responded to the questionnaire during or after the infection. Also, the existence of a postacute COVID-19 syndrome (long-COVID-19) ${ }^{75}$ may impact on dream activity and sleep quality. In this respect, our results should be interpreted with caution since we cannot disentangle whether some effects on oneiric experience were related to the persistence of symptoms or associated with the past experience. Lastly, except for the hypnotics' usage, information on specific types of medications and ongoing treatments was not available. It should be considered that dreams and nightmares can be affected by medication use. ${ }^{76}$ In particular, the pharmacological therapy for COVID-19 patients may include drugs (eg, anodyne, glucocorticoids) that have been reported to affect users' sleep and dreams. ${ }^{16,77}$

Finally, it is worth noting that this current ICOSS protocol focused on the first wave of the pandemic instead of the COVID-19 infection per se. A second survey was done to answer the important questions related to COVID-19 and comorbidities, also addressing the long-COVID-19 issue. ${ }^{78}$ 
Table 3 Ordinal Logistic Regression Model on Nightmare Frequency in COVID-19 Participants (N=499)

\begin{tabular}{|c|c|c|c|c|}
\hline \multirow[t]{2}{*}{ Covariates } & \multirow[t]{2}{*}{$O R$} & \multirow[t]{2}{*}{$p$} & \multicolumn{2}{|c|}{$95 \% \mathrm{Cl}$} \\
\hline & & & Lower Bound & Upper Bound \\
\hline Wellbeing (WHO-5) & 1.00 & 0.459 & 0.99 & I.0I \\
\hline Quality of life & 1.00 & 0.835 & 0.99 & I.0I \\
\hline Quality of health & 1.00 & 0.466 & 0.98 & 1.01 \\
\hline $\begin{array}{l}\text { Anxiety } \\
\text { Yes } \\
\text { No }\end{array}$ & $\begin{array}{l}1.76 \\
\text { Ref }\end{array}$ & 0.018 & 1.10 & 2.81 \\
\hline $\begin{array}{l}\text { Depression } \\
\text { Yes } \\
\text { No }\end{array}$ & $\begin{array}{l}\text { I.4I } \\
\text { Ref }\end{array}$ & 0.157 & 0.88 & 2.28 \\
\hline $\begin{array}{l}\text { Stress } \\
\text { Yes } \\
\text { No }\end{array}$ & $\begin{array}{l}0.82 \\
\text { Ref }\end{array}$ & 0.388 & 0.53 & 1.28 \\
\hline $\begin{array}{l}\text { PTSD (repeated disturbing thoughts/memories) } \\
\text { High risk } \\
\text { Low risk }\end{array}$ & $\begin{array}{l}2.58 \\
\text { Ref }\end{array}$ & $<0.001$ & 1.67 & 3.96 \\
\hline $\begin{array}{l}\text { DRF } \\
\text { Nightly or almost nightly } \\
3-5 \text { nights per week } \\
\text { I-2 nights per week } \\
\text { Less than weekly } \\
\text { Never or less than monthly }\end{array}$ & $\begin{array}{l}19.26 \\
11.54 \\
9.67 \\
2.82 \\
\text { Ref }\end{array}$ & $\begin{array}{l}<0.001 \\
<0.001 \\
<0.001 \\
0.002\end{array}$ & $\begin{array}{l}9.38 \\
6.01 \\
5.11 \\
1.47\end{array}$ & $\begin{array}{r}39.57 \\
22.14 \\
18.31 \\
5.41\end{array}$ \\
\hline $\begin{array}{l}\text { Insomnia } \\
\text { Yes } \\
\text { No }\end{array}$ & $\begin{array}{l}\text { I.63 } \\
\text { Ref }\end{array}$ & 0.039 & 1.02 & 2.60 \\
\hline $\begin{array}{l}\text { Sleep quality } \\
\text { Poor } \\
\text { Good }\end{array}$ & $\begin{array}{c}0.98 \\
\text { Ref }\end{array}$ & 0.935 & 0.65 & 1.48 \\
\hline $\begin{array}{l}\text { Sleep duration } \\
\text { Long } \\
\text { Normal } \\
\text { Short }\end{array}$ & $\begin{array}{r}0.43 \\
0.55 \\
\text { Ref }\end{array}$ & $\begin{array}{l}0.003 \\
0.010\end{array}$ & $\begin{array}{l}0.25 \\
0.35\end{array}$ & $\begin{array}{l}0.75 \\
0.87\end{array}$ \\
\hline $\begin{array}{l}\text { COVID-19 severity } \\
\text { High } \\
\text { Low }\end{array}$ & $\begin{array}{l}1.60 \\
\text { Ref }\end{array}$ & 0.014 & 1.10 & 2.34 \\
\hline $\begin{array}{l}\text { Quarantine duration } \\
>\text { I month } \\
\leq \text { I month }\end{array}$ & $\begin{array}{l}0.95 \\
\text { Ref }\end{array}$ & $0.78 \mathrm{I}$ & 0.66 & 1.37 \\
\hline $\begin{array}{l}\text { Gender } \\
\text { Female } \\
\text { Male }\end{array}$ & $\begin{array}{l}0.75 \\
\text { Ref }\end{array}$ & 0.121 & 0.52 & 1.08 \\
\hline
\end{tabular}

(Continued) 
Table 3 (Continued).

\begin{tabular}{|c|c|c|c|c|}
\hline \multirow[t]{2}{*}{ Covariates } & \multirow[t]{2}{*}{ OR } & \multirow[t]{2}{*}{$p$} & \multicolumn{2}{|c|}{$95 \% \mathrm{Cl}$} \\
\hline & & & Lower Bound & Upper Bound \\
\hline Age & & & & \\
\hline $65+$ & 0.49 & 0.078 & 0.22 & 1.08 \\
\hline $55-64$ & 0.29 & 0.001 & 0.14 & 0.59 \\
\hline $45-54$ & 0.36 & 0.001 & 0.20 & 0.66 \\
\hline $35-44$ & 0.78 & 0.378 & 0.46 & 1.34 \\
\hline $25-34$ & 0.71 & 0.247 & 0.39 & 1.27 \\
\hline$<25$ & Ref & & & \\
\hline
\end{tabular}

Note: Significant values are indicated in bold.

Abbreviations: OR, odd ratio; $\mathrm{Cl}$, confidence interval; PTSD, post-traumatic stress disorder; Ref, reference.

\section{Conclusion}

In summary, we suggest that dream characteristics and, above all, nightmares are possible correlates of COVID-19. Our study is unable to determine if the effects on dreaming activity are explained by the infection per se or by its impact on the general psychological and mental adaptation to the infection. Nevertheless, our work is an important first step in understanding the effects of COVID-19 on dreams and nightmares. Further investigations on longitudinal assessment of changes in sleep and dreaming during pandemic focusing on participants who have had COVID-19 and those with longCOVID-19 are warranted.

\section{Abbreviations}

WHO, World Health Organization; COVID-19, Coronavirus disease 19; DRF, Dream Recall Frequency; Nightmare Frequency, NF; PTSD, Post-Traumatic Stress Disorder; GAD-2, Generalized Anxiety Disorder-2; PHQ-2, Patient Health Questionnaire-2; WHO-5, Well-Being Index; ISI, Insomnia Severity Index; OR, Odds Ratio; CI, Confidence Interval.

\section{Data Sharing Statement}

Due to the nature of this research, participants of this study did not agree for their data to be shared publicly, so supporting data is not available.

\section{Ethics Approval}

All investigators obtained ethical approval or exemptions from their local ethics committee. The study was approved by the Institutional Ethics Committee of the Department of Psychology of "Sapienza" University of Rome and was conducted in accordance with the Declaration of Helsinki (protocol number: 0000836, May 23rd, 2020). Participants voluntarily and anonymously agreed to take part in the survey. General data protection regulations were applied to ensure privacy and confidentiality.

\section{Acknowledgments}

We would like to acknowledge all ICOSS group collaborators.

\section{Author Contributions}

All authors made a significant contribution to the work reported, whether that is in the conception, study design, execution, acquisition of data, analysis and interpretation, or in all these areas; took part in drafting, revising or critically reviewing the article; gave final approval of the version to be published; have agreed on the journal to which the article has been submitted; and agree to be accountable for all aspects of the work. 


\section{Funding}

This research did not receive any specific grant from funding agencies in the public, commercial, or not-for-profit sectors.

\section{Disclosure}

Dr Frances Chung reports personal fees as a consultant from Takeda Pharma, outside the submitted work. Professor Colin A Espie is a Co-Founder and Chief Scientist of, and a shareholder in, Big Health the company that that developed Sleepio a digital CBT intervention for insomnia. Professor Yuichi Inoue reports personal fees in speaking activity from Eisai Co., Ltd., Otsuka Pharmaceutical Co., Ltd, Takeda Pharmaceutical Company Limited, Astellas Pharma Inc., and MSD K.K.; in addition, Professor Yuichi Inoue also reports the Department of Somnology is an endowment department, supported by an unrestricted grant from Phillips Japan, Ltd and KOIKE MEDICAL Co., LTD, outside the submitted work. Dr Kentaro Matsui reports personal fees from Eisai, Meiji Seika Pharma, MSD, Otsuka, Yoshitomi, and Takeda, outside the submitted work. Professor Charles M Morin reports grants from Idorsia, Eisai, l'allemand Health, outside the submitted work; and royalties from Mapi Research Trust. Professor Thomas Penzel reports grants or personal fees for speaker fee and consultation from Cidelec, Phasya, Neuwirth, Jazz, Cerebra, and National Sleep Foundation. He also owns shares from The Siesta Group, outside the submitted work. The authors report no other conflicts of interest in this work.

\section{References}

1. Blanco C, Wall MM, Olfson M. Psychological aspects of the COVID-19 pandemic. J Gen Intern Med. 2020;35:2757-2759. doi:10.1007/s11606020-05955-3

2. Vindegaard N, Benros ME. COVID-19 pandemic and mental health consequences: systematic review of the current evidence. Brain Behav Immun. 2020;89:531-542. doi:10.1016/j.bbi.2020.05.048

3. Deng J, Zhou F, Hou W, et al. The prevalence of depression, anxiety, and sleep disturbances in COVID-19 patients: a meta-analysis. Ann N Y Acad Sci. 2021;1486:90-111. doi:10.1111/nyas.14506

4. Altena E, Baglioni C, Espie CA, et al. Dealing with sleep problems during home confinement due to the COVID-19 outbreak: practical recommendations from a task force of the European CBT-I Academy. J Sleep Res. 2020;29(4):e13052. doi:10.1111/jsr.13052

5. Blume C, Schmidt MH, Cajochen C. Effects of the COVID-19 lockdown on human sleep and rest-activity rhythms. Curr Biol. 2020;30:795-797. doi:10.1016/j.cub.2020.06.021

6. CasaGrande M, Favieri F, Tambelli R, Forte G. The enemy who sealed the world: effects quarantine due to the COVID-19 on sleep quality, anxiety, and psychological distress in the Italian population. Sleep Med. 2020;75:12-20. doi:10.1016/j.sleep.2020.05.011

7. Cellini N, Canale N, Mioni G, Costa S. Changes in sleep pattern, sense of time and digital media use during COVID-19 lockdown in Italy. $J$ Sleep Res. 2020;29:e13074. doi:10.1111/jsr.13074

8. Alfonsi V, Gorgoni M, Scarpelli S, et al. COVID-19 lockdown and poor sleep quality: not the whole story. J Sleep Res. 2021;30:e13368. doi:10.1111/jsr.13368

9. Franceschini C, Musetti A, Zenesini C, et al. Poor quality of sleep and its consequences on mental health during COVID-19 lockdown in Italy. Front Psychol. 2020;11:3072. doi:10.3389/fpsyg.2020.574475

10. Wright KP, Linton SK, Withrow D, et al. Sleep in University students prior to and during COVID-19 stay-at-home orders. Curr Biol. 2020;30:797-798. doi:10.1016/j.cub.2020.06.022

11. Li Y, Qin Q, Sun Q, et al. Insomnia and psychological reactions during the COVID-19 outbreak in China. J Clin Sleep Med. 2020;16:1417-1418. doi: $10.5664 / \mathrm{jcsm} .8524$

12. Jahrami H, BaHammam AS, Bragazzi NL, et al. Sleep problems during the COVID-19 pandemic by population: a systematic review and meta-analysis. J Clin Sleep Med. 2021;17:299-313. doi:10.5664/jcsm.8930

13. Morin CM, Bjorvatn B, Chung F, et al. Insomnia, anxiety, and depression during the COVID-19 pandemic: an International Collaborative Study. Sleep Med. 2021;87:38-45. doi:10.1016/j.sleep.2021.07.035

14. Ferrando M, Bagnasco D, Roustan V, Canonica GW, Braido F, Baiardini I. Sleep complaints and sleep breathing disorders in upper and lower obstructive lung diseases. $J$ Thorac Dis. 2016;8(8):E716-E725. doi:10.21037/jtd.2016.07.82

15. Singh DP, Jamil RT, Mahajan K. Nocturnal cough. In: Cascella M, Rajnik M, Cuomo A, Dulebohn SC, Di Napoli RS, editors. StatPearls. Treasure Island, FL: Stat Pearls Publishing; 2020.

16. Shi L, Lu ZA, Que JY, et al. Prevalence of and risk factors associated with mental health symptoms among the general population in China during the coronavirus disease 2019 pandemic. JAMA Netw Open. 2020;3:e2014053. doi:10.1001/jamanetworkopen.2020.14053

17. Wesselius HM, Van Den Ende ES, Alsma J, et al. Quality and quantity of sleep and factors associated with sleep disturbance in hospitalized patients. JAMA Int Med. 2018;178:1201-1208. doi:10.1001/jamainternmed.2018.2669

18. Barrett D. Dreams about COVID-19 versus normative dreams: trends by gender. Dreaming. 2020;30:216-221. doi:10.1037/drm0000149

19. Pesonen AK, Lipsanen J, Halonen R, et al. Pandemic dreams: network analysis of dream content during the COVID-19 lockdown. Front Psychol. 2020;11:2569. doi:10.3389/fpsyg.2020.573961

20. Iorio I, Sommantico M, Parrello S. Dreaming in the time of COVID-19: a quali-quantitative Italian study. Dreaming. 2020;30:199. doi:10.1037/ drm0000142 
21. Gorgoni M, Scarpelli S, Alfonsi V, et al. Pandemic dreams: quantitative and qualitative features of the oneiric activity during the lockdown due to COVID-19 in Italy. Sleep Med. 2021;81:20-32. doi:10.1016/j.sleep.2021.02.006

22. Fränkl EC, Scarpelli S, Nadorff MR, et al. How our dreams changed during the COVID-19 pandemic: effects and correlates of dream recall frequency during the COVID-19 pandemic: a Multinational Study on 19,355 adults. Nat Sci Sleep. 2021;13:1573-1591. doi:10.2147/NSS. S324142

23. Mota NB, Weissheimer J, Ribeiro M, et al. Dreaming during the COVID-19 pandemic: computational assessment of dream reports reveals mental suffering related to fear of contagion. PLoS One. 2020;15:e0242903. doi:10.1371/journal.pone.0242903

24. Scarpelli S, Alfonsi V, Mangiaruga A, et al. Pandemic nightmares: effects on dream activity of the COVID-19 lockdown in Italy. $J$ Sleep Res. 2021;30:e13300. doi:10.1111/jsr.13300

25. Scarpelli S, Gorgoni M, Alfonsi V, et al. The impact of the end of COVID confinement on pandemic dreams, as assessed by a weekly sleep diary: a longitudinal investigation in Italy. J Sleep Res. 2021:e13429. doi:10.1111/jsr.13429

26. Schredl M, Reinhard I. Gender differences in nightmare frequency: a meta-analysis. Sleep Med Rev. 2011;15:115-121. doi:10.1016/j. smrv.2010.06.002

27. Schredl M, Bulkeley K. Dreaming and the COVID-19 pandemic: a survey in a US sample. Dreaming. 2020;30:189. doi:10.1037/drm0000146

28. MacKay C, DeCicco TL. Pandemic dreaming: the effect of COVID-19 on dream imagery, a pilot study. Dreaming. 2020;30:222. doi:10.1037/ drm0000148

29. Wang H, Xia Q, Xiong Z, et al. The psychological distress and coping styles in the early stages of the 2019 coronavirus disease (COVID-19) epidemic in the general mainland Chinese population: a web-based survey. PLoS One. 2020;15:e0233410. doi:10.1371/journal.pone.0233410

30. Becker RC. Anticipating the long-term cardiovascular effects of COVID-19. J Thromb Thrombolysis. 2020;50:512-524. doi:10.1007/s11239-02002266-6

31. Del Rio C, Collins LF, Malani P. Long-term health consequences of COVID-19. JAMA. 2020;324:1723-1724. doi:10.1001/jama.2020.19719

32. Heneka MT, Golenbock D, Latz E, Morgan D, Brown R. Immediate and long-term consequences of COVID-19 infections for the development of neurological disease. Alzheimers Res Ther. 2020;12:1-3. doi:10.1186/s13195-020-00640-3

33. Ritchie K, Chan D, Watermeyer T. The cognitive consequences of the COVID-19 epidemic: collateral damage? Brain Commun. $2020 ; 2$ :fcaa069. doi:10.1093/braincomms/fcaa069

34. Iqbal SZ, Li B, Onigu-Otito E, Faraz Naqui M, Shah AA. The long-term mental health effects of COVID-19. Psychiatr Ann. 2020;50:522-525. doi:10.3928/00485713-20201103-01

35. Nadorff MR, Nazem S, Fiske A. Insomnia symptoms, nightmares, and suicidal ideation in a college student sample. Sleep. 2011;34:93-98. doi:10.1093/sleep/34.1.93

36. Selby EA, Ribeiro JD, Joiner TE Jr. What dreams may come: emotional cascades and nightmares in borderline personality disorder. Dreaming. 2013;23:126-144. doi:10.1037/a0032208

37. Tanskanen A, Tuomilehto J, Viinamäki H, Vartiainen E, Lehtonen J, Puska P. Nightmares as predictors of suicide. Sleep. 2001;24:845-848. doi:10.1093/sleep/24.7.845

38. Li SX, Lam SP, Mandy WM, Zhang J, Wing YK. Nocturnal sleep disturbances as a predictor of suicide attempts among psychiatric outpatients: a clinical, epidemiologic, prospective study. J Clin Psychiatry. 2010;71:1440-1446. doi:10.4088/JCP.09m05661gry

39. Sjöström N, Hetta J, Waern M. Persistent nightmares are associated with repeat suicide attempt: a prospective study. Psychiatry Res. 2009;170:208-211. doi:10.1016/j.psychres.2008.09.006

40. Nadorff MR, Nazem S, Fiske A. Insomnia symptoms, nightmares, and suicide risk: duration of sleep disturbance matters. Suicide Life Threat Behav. 2013;43:139-149. doi:10.1111/sltb.12003

41. Partinen M, Bjorvatn B, Holzinger B, Chung F, Penzel T, Espie CA; ICOSS-collaboration group. Sleep and circadian problems during the coronavirus disease 2019 (COVID-19) pandemic: the International COVID-19 Sleep Study (ICOSS). J Sleep Res. 2021;30:e13206. doi:10.1111/ jsr.13206

42. Chung F, Yegneswaran B, Liao P, et al. STOP questionnaire: a tool to screen patients for obstructive sleep apnea. Anesthesiology. 2008;108:812-821. doi:10.1097/ALN.0b013e31816d83e4

43. Kroenke K, Spitzer RL, Williams JBW, Löwe B. An ultra-brief screening scale for anxiety and depression: the PHQ-4. Psychosomatics. 2009;50:613-621. doi:10.1176/appi.psy.50.6.613

44. Elo AL, Leppänen A, Jahkola A. Validity of a single-item measure of stress symptoms. Scand J Work Environ Health. 2003;29:444-451. doi:10.5271/sjweh.752

45. Lang AJ, Wilkins K, Roy-Byrne PP, et al. Abbreviated PTSD checklist (PCL) as a guide to clinical response. Gen Hosp Psychiatry. 2012;34:332-338. doi:10.1016/j.genhosppsych.2012.02.003

46. Topp CW, Østergaard SD, Søndergaard S, Bech P. The WHO-5 wellbeing index: a systematic review of the literature. Psychother Psychosom. 2015;84:167-176. doi:10.1159/000376585

47. de Boer AGEM, van Lanschot JJB, Stalmeier PFM, et al. Is a single-item visual analogue scale as valid, reliable and responsive as multi-item scales in measuring quality of life? Qual Life Res. 2004;13:311-320. doi:10.1023/B:QURE.0000018499.64574.1f

48. Partinen M, Gislason T. Basic Nordic Sleep Questionnaire (BNSQ): a quantitated measure of subjective sleep complaints. $J$ Sleep Res. 1995;4:150-155. doi:10.1111/j.1365-2869.1995.tb00205.x

49. Morin CM, Belleville G, Bélanger L, Ivers H. The Insomnia Severity Index: psychometric indicators to detect insomnia cases and evaluate treatment response. Sleep. 2011;34:601-608. doi:10.1093/sleep/34.5.601

50. Aeschbach D, Matthews JR, Postolache TT, Jackson MA, Giesen HA, Wehr TA. Two circadian rhythms in the human electroencephalogram during wakefulness. Am J Physiol Reg I. 1999;277:R1771-R1779. doi:10.1152/ajpregu.1999.277.6.R1771

51. Aeschbach D, Sher L, Postolache TT, Matthews JR, Jackson MA, Wehr TA. A longer biological night in long sleepers than in short sleepers. J Clin Endocrinol Metab. 2003;88:26-30. doi:10.1210/jc.2002-020827

52. Neylan TC, Marmar CR, Metzler TJ, et al. Sleep disturbances in the Vietnam generation: findings from a nationally representative sample of male Vietnam veterans. Am J Psychiatry. 1998;155:929-933. doi:10.1176/ajp.155.7.929

53. Tempesta D, Curcio G, De Gennaro L, Ferrara M. Long-term impact of earthquakes on sleep quality. PLoS One. 2013;8:e55936. doi:10.1371/ journal.pone. 0055936 
54. Mian A, Khan S. Coronavirus: the spread of misinformation. BMC Med. 2020;18:1-2. doi:10.1186/s12916-020-01556-3

55. Poyraz BÇ, Poyraz CA, Olgun Y, et al. Psychiatric morbidity and protracted symptoms after COVID-19. Psychiatry Res. 2021;295:113604. doi:10.1016/j.psychres.2020.113604

56. American Psychiatric Association. Diagnostic and Statistical Manual of Mental Disorders: DSM-5. 4th ed. Arlington, VA: American Psychiatric Publishing; 2013.

57. Dubey S, Biswas P, Ghosh R, et al. Psychosocial impact of COVID-19. Diabetes Metab Syndr. 2020;14:779-788. doi:10.1016/j.dsx.2020.05.035

58. Levin R, Nielsen T. Nightmares, bad dreams, and emotion dysregulation: a review and new neurocognitive model of dreaming. Curr Dir Psychol Sci. 2009;18:84-88. doi:10.1111/j.1467-8721.2009.01614.x

59. Schredl M. Dreams in patients with sleep disorders. Sleep Med Rev. 2009;13:215-221. doi:10.1016/j.smrv.2008.06.002

60. D'Atri A, Scarpelli S, Schiappa C, et al. Cortical activation during sleep predicts dream experience in narcolepsy. Ann Clin Transl Neur. 2019;6:445-455. doi:10.1002/acn3.718

61. van Wyk M, Solms ML, Lipinska G. Increased awakenings from non-rapid eye movement sleep explains differences in dream recall frequency in healthy high and low recallers. Front Hum Neurosci. 2019;13:370. doi:10.3389/fnhum.2019.00370

62. Scarpelli S, Bartolacci C, D'Atri A, et al. Electrophysiological correlates of dream recall during REM sleep: evidence from multiple awakenings and within-subjects design. Nat Sci Sleep. 2020;12:1043. doi:10.2147/NSS.S279786

63. Newell SA, Padamadan H, Drake ME Jr. Neurophysiologic studies in nightmare sufferers. Clin Electroencephal. 1992;23:203-206. doi:10.1177/ 155005949202300410

64. Simor P, Bódizs R, Horváth K, Ferri R. Disturbed dreaming and the instability of sleep: altered nonrapid eye movement sleep microstructure in individuals with frequent nightmares as revealed by the cyclic alternating pattern. Sleep. 2013;36:413-419. doi:10.5665/sleep.2462

65. Van Someren EJ. Brain mechanisms of insomnia: new perspectives on causes and consequences. Physiol Rev. 2021;101:995-1046. doi:10.1152/ physrev.00046.2019

66. Nakajima S, Inoue Y, Sasai T, et al. Impact of frequency of nightmares comorbid with insomnia on depression in Japanese rural community residents: a cross-sectional study. Sleep Med. 2014;15:371-374. doi:10.1016/j.sleep.2013.11.785

67. Scarpelli S, Bartolacci C, D’Atri A, Gorgoni M, De Gennaro L. Mental sleep activity and disturbing dreams in the lifespan. Int J Environ Res Public Health. 2019;16:3658. doi:10.3390/ijerph16193658

68. Mangiaruga A, Scarpelli S, Bartolacci C, De Gennaro L. Spotlight on dream recall: the ages of dreams. Nat Sci Sleep. 2018;10:1. doi:10.2147/NSS. S135762

69. Strunz F. Dreams in the elderly: contents and clinical usefulness. Gesundheitswesen. 1993;55:595-601.

70. Belicki K. Nightmare frequency versus nightmare distress: relation to psychopathology and cognitive style. J Abnorm Psychol. $1992 ; 101: 592-597$. doi:10.1037/0021-843X.101.3.592

71. Chivers L, Blagrove M. Nightmare frequency, personality and acute psychopathology. Pers Individ Diff. 1999;27:843-851. doi:10.1016/S01918869(99)00033-1

72. Schredl M. Effects of state and trait factors on nightmare frequency. Eur Arch Psychiatry Clin Neurosci. 2003;253:241-247. doi:10.1007/s00406003-0438-1

73. Zadra A, Robert G. Dream recall frequency: impact of prospective measures and motivational factors. Conscious Cogn. 2012;21:1695-1702. doi:10.1016/j.concog.2012.08.011

74. Palagini L, Rosenlicht N. Sleep, dreaming, and mental health: a review of historical and neurobiological perspectives. Sleep Med Rev. 2011;15:179-186. doi:10.1016/j.smrv.2010.07.003

75. Al-Jahdhami I, Al-Naamani K, Al-Mawali A. The post-acute COVID-19 syndrome (long COVID). Oman Med J. 2021;36:e220. doi:10.5001/ omj.2021.91

76. Siclari F, Valli K, Arnulf I. Dreams and nightmares in healthy adults and in patients with sleep and neurological disorders. Lancet Neurol. 2020;19:849-859. doi:10.1016/S1474-4422(20)30275-1

77. Szmyd B, Rogut M, Białasiewicz P, Gabryelska A. The impact of glucocorticoids and statins on sleep quality. Sleep Med Rev. 2021;55:101380. doi:10.1016/j.smrv.2020.101380

78. Merikanto I, Dauvilliers Y, Chung F, et al.; ICOSS members. Disturbances in sleep, circadian rhythms, and daytime functioning in relation to coronavirus infection and long-COVID - a multinational ICOSS study. J Sleep Res. 2021. doi:10.1111/jsr.13542

\section{Publish your work in this journal}

Nature and Science of Sleep is an international, peer-reviewed, open access journal covering all aspects of sleep science and sleep medicine, including the neurophysiology and functions of sleep, the genetics of sleep, sleep and society, biological rhythms, dreaming, sleep disorders and therapy, and strategies to optimize healthy sleep. The manuscript management system is completely online and includes a very quick and fair peer-review system, which is all easy to use. Visit http://www.dovepress.com/testimonials.php to read real quotes from published authors. 